\title{
A NOTE ON TRIPLE SYSTEMS AND TOTALLY GEODESIC SUBMANIFOLDS IN A HOMOGENEOUS SPACE
}

\author{
ARTHUR A. SAGLE
}

1. Introduction. In the study of nonassociative algebras various "triple systems" frequently arise from the associator function and other multilinear objects. In particular Lie triple systems arise in the study of Jordan algebras and a generalization of a Lie triple system arises in Malcev algebras. Lie triple systems also are used to study totally geodesic submanifolds of a Riemannian symmetric space. We shall show how a generalization of Lie triple systems also arises from the study of curvature and geodesics of a torsion free connexion on a manifold and bring out the relation of this to various nonassociative algebras.

To establish notation we briefly review connexions, curvature and the second fundamental form in sections 2 and 3 and explicitly indicate how the results of [13] can be generalized to a manifold and to the study of the second fundamental form. In section 4 we use the results of [6] on reductive homogeneous spaces to show the relationships between certain nonassociative algebras, generalization of Lie triple systems and totally geodesic submanifolds. In section 5 we shall show that the Lie algebra of the holonomy group and the curvature formula for a reductive homogeneous space are well behaved in terms of certain nonassociative algebras. Also we shall give examples for which the curvature formula and the totally geodesic submanifolds are easy to determine by these algebras.

2. Basics. To establish notation we review some basic facts about covariant differentiation, torsion and curvature as given in $[1,3]$. Let $M$ be a $C^{\infty}$ manifold and let $D$ be a covariant differentiation operator (i.e. connexion) defined on $M$. Thus for each pair of $C^{\infty}$ vector fields defined on a suitable domain $A \subset M$ [3] we have a $C^{\infty}$ vector field $D_{X} Y=D(X, Y)$ with domain $A$ such that if $Z$ is a $C^{\infty}$ vector field on $A$ and $f$ a $C^{\infty}$ real valued function on $A$, then $D$ satisfies

Received February 7, 1967.

This research was supported in part by N.S.F. Grant GP-1453 


$$
\begin{aligned}
& D(X, Y+Z)=D(X, Y)+D(X, Z), \\
& D(X+Y, Z)=D(X, Z)+D(Y, Z), \\
& D(f X, Y)=f D(X, Y), \\
& D(X, f Y)=(X f) Y+f D(X, Y) .
\end{aligned}
$$

Next we define the torsion and curvature tensors of $D$ by

$$
\text { Tor }(X, Y)=D(X, Y)-D(Y, X)-[X, Y] \text {, }
$$

where $[A, B]=A B-B A$. Thus the torsion tensor, Tor, assigns to each pair of $C^{\infty}$ vector fields $X$ and $Y$ with domain $A$ a $C^{\infty}$ vector field Tor $(X, Y)$ with domain $A$. Note that $\operatorname{Tor}(X+Y, Z)=\operatorname{Tor}(X, Z)+\operatorname{Tor}(Y, Z)$, $\operatorname{Tor}(f X, Y)=f \operatorname{Tor}(X, Y)$ and $\operatorname{Tor}(X, Y)=-\operatorname{Tor}(Y, X)$. We define the curvature tensor, $R$, for $C^{\infty}$ vector fields $X, Y$ and $Z$ with domain $A$ by

$$
\begin{aligned}
R(X, Y) Z= & D(X, D(Y, Z))-D(Y, D(X, Z)) \\
& -D([X, Y], Z) \\
\equiv & {\left[D_{X}, D_{Y}\right] Z-D_{[X, Y]} Z . }
\end{aligned}
$$

In particular this says that the curvature measures the failure of the map $X \rightarrow D_{X}$ to be a Lie algebra homomorphism. If $f$ is a $C^{\infty}$ real valued function which is defined on $A$, then $R$ satisfies $R(f X, Y) Z=f R(X, Y) Z=$ $R(X, Y)(f Z), R(X, Y) Z=-R(Y, X) Z$ and $R(X, Y) Z$ is additive in each of its variables.

Next, an abstract Lie triple system has been defined [13] to be a vector space $\vee$ over a field $F$ with an operation $[X, Y, Z]$ defined on $\vee \times \vee \times \vee$ into $V$ satisfying

(2. 1) $[X, Y, Z]$ is trilinear over $F$,

(2. 2) $[X, Y, Z]=-[Y, X, Z]$,

(2. 3) $[X, Y, Z]+[Y, Z, X]+[Z, X, Y]=0$.

These are identities (i), (ii) and (v) of a Lie triple system (L.t.s.), see [5]. In particular if we set $[X, Y, Z]=R(X, Y) Z$ for $C^{\infty}$ vector fields $X, Y, Z$ on $M$, we see that $[X, Y, Z]$ satisfies (2.1) and (2.2) over the commutative algebra $F(M)$ of $C^{\infty}$ functions on $M$.

One of the properties of a semi-Riemannian connexion on a manifold $M$ we want is that $\operatorname{Tor}(X, Y)=0$ for vector fields $X$ and $Y$. In this case we have the 1 st and 2 nd Bianchi identities which are respectively $[X, Y, Z]+$ 
$[Y, Z, X]+[Z, X, Y]=0 \quad$ and $\quad\left(D_{X} R\right)(Y, Z)+\left(D_{Y} R\right)(Z, X)+\left(D_{Z} R\right)(X, Y)=0$, where $D_{U} R$ denotes covariant differentation of $R$ relative to $U$. The 1st Bianachi is identity (2.3) above. Thus if $\mathfrak{X}(M)$ denotes the set of all $C^{\infty}$ vector fields on $M$ and Tor $(X, Y)=0$ for all $X, Y$ in $\mathfrak{X}(M)$, then $\mathfrak{X}(M)$ is an abstract L.t.s over $F(M)$ relative to the operation $[X, Y, Z]=R(X, Y) Z$. This definition generalizes to any left module over a commutative ring $\Phi$ with identity.

The study of a L.t.s $\mathfrak{T}$ involves an imbedding of $\mathfrak{T}$ into a Lie algebra $\mathfrak{L}$ which is roughly given by $\mathfrak{L}=\mathfrak{T}+[\mathfrak{T} \mathfrak{I}]$ where $[U V]$ denotes the Lie algebra multiplication [5]. It was shown in [13] that an abstract L.t.s can be imbedded in a Lie algebra as follows if it satisfies an additional identity. Let $\mathfrak{X}(M)$ be the left $F(M)$-module as above with a trilinear operation $[X, Y, Z]$ for $X, Y, Z$ in $\mathfrak{X}(M)$, satisfying (2.1)-(2.3). Assume $\mathfrak{X}(M)$ also

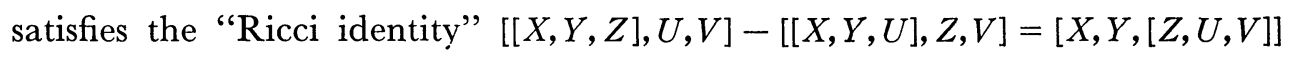
$-[Z, U,[X, Y, V]]$, then if we set $H(X, Y): X(M) \rightarrow X(M): Z \rightarrow[X, Y, Z]$ and $\mathfrak{Y}=\{H(X, Y): X, Y$ in $\mathfrak{X}(M)\}$, the Ricci identity together with the usual vector space operations show $\mathfrak{S}$ is a (left) Lie ring over $F(M)$ under commutation. Consequently $\mathfrak{S}=\mathfrak{X}(M) \oplus \mathfrak{S}$ can be made naturally into a Lie ring with the properties similar to the Lie algebras used to study a symmetric space $[6,1]$; that is, if $\mathfrak{m}=\mathfrak{X}(M)$, then $[\mathfrak{m} \mathfrak{g}] \subset \mathfrak{m}$ and $[\mathfrak{m} \mathfrak{m}] \subset \mathfrak{g}$ so that $\mathfrak{m}$ with the operation $[X, Y, Z]$ is a L.t.s. The algebra of a symmetric pair $(\mathfrak{g}, \mathfrak{S})$ with decomposition $\mathfrak{g}=\mathfrak{m}+\mathfrak{S}$ will be generalized in section 5 to a "reductive pair" $(\mathfrak{g}, \mathfrak{g})$.

Let $M$ be a $C^{\infty}$ semi-Riemannian manifold with nondegenerate metric tensor $<,>$; that is, for each $m$ in $M,<,>$ induces a nondegenerate bilinear form $<,>_{m}$ on each tangent space $M_{m}$. Now there exists a unique connexion $D$ on the semi-Riemannian manifold $M$ such that for $X, Y, Z$ in $\mathfrak{X}(M)$,

(2. 4) $0=\operatorname{Tor}(X, Y)=D(X, Y)-D(Y, X)-[X, Y]$,

(2. 5) $Z<X, Y>=<D(Z, X), Y>+<X, D(Z, Y)>$;

that is, $D$ preserves the metric tensor under parallel translation [3]. We shall assume in this paper that the connexion $D$ on a semi-Riemannian manifold satisfy (2.4) and (2.5). Thus since $\operatorname{Tor}(X, Y)=0$ we see that $\mathfrak{X}(M)$ is an abstract L.t.s. with other relations involving the metric tensor [3]. 
3. Totally geodesic submanifolds. Let $M$ be a $C^{\infty}$ semi-Riemannian manifold and let $M^{\prime}$ be a nonsingular submanifold of $M$; that is, $M^{\prime}$ is a submanifold of $M$ such that the metric tensor of $M$ when restricted to the tangent space $M_{m}^{\prime}$ for all $m \in M^{\prime}$ is nondegenerate on $M_{m}^{\prime}$. The semiRiemannian connexion $D$ on $M$ induces a semi-Riemannian connexion $D^{\prime}$ on $M^{\prime}$ as follows [2,3]. Let $M^{\prime}$ be a nonsingular submanifold of $M$, then for $C^{\infty}$ vector fields $X$ and $Y$ with domain $A \subset M^{\prime}$ and tangent to $M^{\prime}$, define $D^{\prime}(X, Y)$ and $V(X, Y)$ by extending $X, Y$ to a vector field on an open set of $M$ containing $A$ and by decomposing $D(X, Y)$ into its unique tangential and normal components, respectively, relative to the metric tensor on $M$. Thus

$$
D(X, Y)=D^{\prime}(X, Y)+V(X, Y)
$$

Then $D^{\prime}$ is a connexion on $M^{\prime}$ which satisfies (2.4) and (2.5) for $M^{\prime}$ and $V$ is a symmetric vector valued 2-covariant $C^{\infty}$ tensor called the second fundamental form tensor.

Following the notation in [2] we decompose any vector field $W$ on $M^{\prime}$ into its tangent and normal components and write $W=\tan W+$ nor $W$. Thus for $C^{\infty}$ vector fields $X, Y, Z \in \mathfrak{X}\left(M^{\prime}\right)$ on $M^{\prime}$ we have

$$
\tan R(X, Y) Z=R^{\prime}(X, Y) Z+\tan [D(X, V(Y, Z))-D(Y, V(X, Z))]
$$

where $R$ (resp. $R^{\prime}$ ) is the curvature of $M$ (resp. $M^{\prime}$ ). As in [2] this formula can be simplified as follows. The nonsingular submanifold $M^{\prime}$ is framed in $M$ if one can choose $n-k=d$ unit mutally orthogonal $C^{\infty}$ vector fields on $M^{\prime}$ which are normal to $M^{\prime}\left(n=\operatorname{dim} . M, k=\operatorname{dim} . M^{\prime}\right) . \quad M^{\prime}$ can always be framed locally in $M$. Thus assume $M^{\prime}$ is framed in $M$ and let $N_{1}, \cdots, N_{d}$ be the set of normal vectors. Then define $d$ " 2 nd fundamental forms" $B_{i}$ on $M^{\prime}$ by $V(X, Y)=\sum_{i=1}^{d} B_{i}(X, Y) N_{i}$. Each $B_{i}$ is a real valued symmetric $C^{\infty}$ 2-covariant tensor on $M^{\prime}$ and depends on the choice of $N_{1}, \cdots, N_{d}$. Next for each $i$ define the Weingarten maps $L_{i}$ to be the $F\left(M^{\prime}\right)$-linear transformation on $\mathfrak{X}\left(M^{\prime}\right)$ given by $r_{i} L_{i}(X)=\tan D\left(X, N_{i}\right)$ where $r_{i}=\left\langle N_{i}, N_{i}\right\rangle= \pm 1$, see $[2,3]$. When evaluated at $m$ in $M^{\prime}, L_{i}$ becomes a linear transformation on the tangent space $M_{m}^{\prime}$.

Using the above notation we have from [2] that $\left\langle V(X, Y), N_{i}\right\rangle$ $=-r_{i}<Y, L_{i} X>$ and $B_{i}(X, Y)=-<X, L_{i} Y>=-<L_{i} X, Y>$ which shows 
each $L_{i}$ is self adjoint. Also $V(X, Y)=-\sum_{i=1}^{a}<X, L_{i} Y>N_{i}$. The Gauss curvature equation (3.2) now becomes

(3. $\left.2^{\prime}\right) \tan R(X, Y) Z=R^{\prime}(X, Y) Z+\sum_{i=1}^{a} r_{i}\left(<L_{i} X, Z>L_{i} Y-<L_{i} Y, Z>L_{i} X\right)$.

From the various equations we see that $\tan R-R^{\prime}$ also yields an abstract L.t.s. structure on $\mathfrak{X}\left(M^{\prime}\right)$ given by

$$
\begin{aligned}
{[X, Y, Z] } & =\tan R(X, Y) Z-R^{\prime}(X, Y) Z \\
& =\sum_{i=1}^{d} r_{i}\left(<L_{i} X, Z>L_{i} Y-<L_{i} Y, Z>L_{i} X\right) . \\
& =\sum_{i=1}^{d}[X, Y, Z]_{i}
\end{aligned}
$$

where each $[X, Y, Z]_{i}=r_{i}\left(<L_{i} X, Z>L_{i} Y-<L_{i} Y, Z>L_{i} X\right)$ yields an abstract Lie triple "subsystem" from which the abstract L.t.s., $[X, Y, Z]$, is constructed.

Let $\sigma$ be a $C^{\infty}$ curve in the $C^{\infty}$ semi-Riemannian manifold $M$ with tangent vector field $T$. $\sigma$ is a geodesic if $D(T, T)=0$ on $\sigma$. A nonsingular submanifold $M^{\prime}$ of $M$ is totally geodesic at a point $m$ in $M^{\prime}$ if for every $X$ in $M_{m}^{\prime}$, the geodesic $\sigma(t)$ of $M$ passing through $m$ and with tangent vector $X$ lies in $M^{\prime}$ for small value of the parameter $t$. If $M^{\prime}$ is totally geodesic at every point of $M^{\prime}$, it is called a totally geodesic submanifold of $M$. From this we have that if $M^{\prime}$ is totally geodesic at a point, then the 2nd fundamental form tensor vanishes at that point; thus $M^{\prime}$ is a totally geodesic submanifold yields $V(X, Y)=0$ for all $X, Y$ in $\mathfrak{X}\left(M^{\prime}\right)$. Using this with (3.1) and (3.2) we easily obtain the following standard result [12].

Proposition. Let $M^{\prime}$ be a nonsingular $C^{\infty}$ submanifold of a semi-Riemannian $C^{\infty}$ manifold $M$, then the following are equivalent

(i) $M^{\prime}$ is totally geodesic,

(ii) geodesics in $M^{\prime}$ are geodesics in $M$,

(iii) parallel translation in $M^{\prime}$ and $M$ are the same.

Any one of the above imply

(iv) if $X, Y, Z$ are in $\mathfrak{X}\left(M^{\prime}\right)$, then $[X, Y, Z]=R(X, Y) Z$ is in $\mathfrak{X}\left(M^{\prime}\right)$.

Thus evaluating at a given point $m$ in $M^{\prime}$, each tangent space $M_{m}^{\prime}$ becomes an abstract L.t.s.

4. Totally geodesic submanifolds of a reductive homogeneous space. Let $G$ be a connected Lie group and $H$ a closed Lie subgroup so that 
the homogeneous space $M=G / H$ is reductive; that is, if $\mathfrak{g}$ (resp. $\mathfrak{h}$ ) is the Lie algebra of $G$ (resp. $H$ ), there exists a subspace $\mathfrak{m}$ of $\mathfrak{g}$ such that $\mathfrak{g}=\mathfrak{m}+\mathfrak{h}$ (subspace direct sum) where $\operatorname{ad}(H) \mathfrak{m} \subset \mathfrak{m}$; that is, [ $\mathfrak{m} \mathfrak{h}] \subset \mathfrak{m}$ if $H$ is connected where $[a b]$ denotes Lie algebra multiplication in $g[6]$. Now there exists a neighborhood $V$ of the identity element $e$ in $G$ such that $V=N \times K$ where $K$ is contained in the identity component of $H$ [6]. Also if $\pi: G \rightarrow G / H$ is the canonical projection, then $\pi$ is a differentiable homeomorphism of $N$ onto a neighborhood $N^{*}$ of $p_{0}=H$ in $G / H$ and $N^{*}=\{c H: c$ in $N\}$.

Identifying the tangent space, $M_{p_{0}}$, of $p_{0}$ in $M$ with $\mathfrak{m}$ we define for each $X$ in $\mathfrak{m}$ a vector field $X^{*}$ with domain $A=N^{*}$ by

$$
\left(X^{*}\right)_{\tau(c) \cdot p_{0}}=d \tau(c) p_{0} X
$$

where $c$ is in $N, \tau(c) \cdot p_{0}=c H$ and $\left(X^{*}\right) p_{0}=X$ in $\mathfrak{m}$. With this notation we now state the main theorem for invariant affine connexions on $M=G / H$; an invariant affine connexion is an affine connexion which is invariant by the mappings $\tau(a): M \rightarrow M: x H \rightarrow a x H$, for all $a$ in $G$ [6]

Theorem. Let $G / H$ be a reductive homogeneous space with a fixed Lie algebra decomposition $\mathfrak{g}=\mathfrak{m}+\mathfrak{h}$ with ad $(H) \mathfrak{m} \subset \mathfrak{m}$. Then there exists a one-to-one correspondence between the set of all invariant affine connections on $G / H$ and the set of all bilinear functions $\alpha: \mathfrak{m} \times \mathfrak{m} \rightarrow \mathfrak{m}$ which are ad(H)-invariant; that is, $a d(h) \alpha(X, Y)=\alpha(a d(h) X, a d(h) Y)$ for $X, Y$ in $\mathfrak{m}$ and $h$ in $\mathfrak{h}$. The correspondence is given by

$$
\alpha(X, Y)=D\left(X^{*}, Y^{*}\right) p_{0} .
$$

Thus corresponding to an invariant affine connection we can associate a nonassociative algebra multiplication $\alpha(X, Y)$ on $\mathfrak{m} ; \alpha$ is called a connexion function [6]. Now for a fixed decomposition $\mathfrak{g}=\mathfrak{m} \dot{+} \mathfrak{h}$ with $[\mathfrak{m} \mathfrak{h}] \subset \mathfrak{m}$ we can define a multiplication, $X Y$, on $\mathfrak{m}$ as follows; for $X, Y$ in $\mathfrak{m}$, let $[X Y]$ $=X Y+h(X, Y)$ where $X Y=[X Y]_{\mathfrak{m}}$ (resp. $\left.h(X, Y)=[X Y]_{\mathfrak{h}}\right)$ is the projection of $[X Y]$ in $\mathfrak{g}$ into $\mathfrak{m}$ (resp. $\mathfrak{h}$ ). The identities which $X, Y, Z$ in $\mathfrak{m}$ and $h, h(X, Y)$ in $\mathfrak{h}$ satisfy are obtained from the Lie algebra identities of $\mathfrak{g}=\mathfrak{m}+\mathfrak{h}$ and these are

$$
\begin{array}{ll}
\text { (4. 1) } & X Y=-Y X \quad \text { (bilinear) } \\
\text { (4. 2) } & h(X, Y)=-h(Y, X) \quad \text { (bilinear) } \\
\text { (4. 3) } & {[Z h(X, Y)]+[X h(Y, Z)]+[Y h(Z, X)]=J(X, Y, Z) \equiv(X Y) Z} \\
& +(Y Z) X+(Z X) Y
\end{array}
$$


(4. 4)

$$
\begin{array}{ll}
\text { (4. 4) } & h(X Y, Z)+h(Y Z, X)+h(Z X, Y)=0 \\
\text { (4. 5) } & {[h(X, Y) h]=h([X h], Y)+h(X,[Y h])}
\end{array}
$$$$
\text { (4. 6) }\left[\begin{array}{ll}
h & X Y
\end{array}\right]=\left[\begin{array}{ll}
h & X
\end{array}\right] Y+X\left[\begin{array}{ll}
h & Y
\end{array}\right] \text {. }
$$

In particular (4.6) says that the mapping $D(h)=a d_{\mathfrak{m}} h: \mathfrak{m} \rightarrow \mathfrak{m}$ : $X \rightarrow\left[\begin{array}{ll}h & X\end{array}\right]$ is a derivation of the algebra $\mathfrak{m}$.

If the reductive space $M=G / H$ is a semi-Riemannian manifold, then the metric tensor, $\langle X, Y\rangle$, on $M$ is obtained by translating a nondegenerate bilinear form, $\langle X, Y\rangle_{0}$, on $\mathfrak{m}=M_{p_{0}}$ by elements in $G$ to the appropriate tangent space $M_{\tau(c) \cdot p_{0}}$. In particular the form $<X, Y>_{0}$ on $\mathfrak{m}$ is $a d(H)$ invariant; thus we have $0=\left\langle[X h], Y>_{0}+<X,[Y h]>_{0}\right.$ for $X, Y$ in $\mathfrak{m}$ and $h$ in $\mathfrak{h}$. It is shown in [6] that if a connexion, $D$, on $M=G / H$ satisfies (2. 4) and (2.5) for the above metric tensor and if the 1-parameter subgroup $x(t)$ of $G$ generated by an element $X$ in $\mathfrak{m}$ projects by $\pi: G \rightarrow G / H: x(t)$ $\rightarrow x^{*}(t)$ so that $x^{*}(t)$ is a geodesic in $G / H$, then the connexion function for $D$ is given by $\alpha(X, Y)=\frac{1}{2} X Y\left(=\frac{1}{2}[X Y]_{\mathfrak{m}}\right)$ for $X, Y$ in $\mathfrak{m}$. In this case it is also shown that $\mathfrak{m}$ satisfies $\left\langle X Y, Z>_{0}=\left\langle X, Y Z>_{0}\right.\right.$; thus $\mathfrak{m}$ is an anti-commutative algebra with a nondegenerate invariant form and multiplication in $\mathfrak{m}$ is given by the (fixed) decomposition $\mathfrak{g}=\mathfrak{m}+\mathfrak{h}$. Also according to [6] the curvature formula for $M=G / H$ evaluated at $p_{0}$ in $M$ is given by

$$
R(X, Y) Z=\frac{1}{4} X(Y Z)-\frac{1}{4} Y(X Z)-\frac{1}{2}(X Y) Z-[h(X, Y) Z]
$$

for $X, Y, Z$ in $\mathfrak{m}$. Note that (4.7) shows a subalgebra $\mathfrak{m}^{\prime}$ of $\mathfrak{m}$ (with

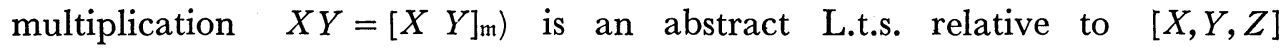
$=R(X, Y) Z$ if and only if $\mathfrak{m}^{\prime}$ is $h\left(\mathfrak{m}^{\prime}, \mathfrak{m}^{\prime}\right)$-invariant; that is, invariant under the set of linear transformations $a d \mathfrak{m} h\left(\mathfrak{m}^{\prime} \mathfrak{m}^{\prime}\right)=\left\{a d_{\mathfrak{m}} h(X, Y): X, Y\right.$ in $\left.\mathfrak{m}^{\prime}\right\}$.

From $[6,1]$ a symmetric space $M=G / H$ is reductive with decomposition $\mathfrak{g}=\mathfrak{m}+\mathfrak{h}$ where for $X, Y$ in $\mathfrak{m}$ we have $X Y=[X Y]_{\mathfrak{m}}=0$. Using this and the formula for the curvature of a Riemannian globally symmetric space [1], we see that the following generalizes the results on totally geodesic submanifolds and Lie triple systems.

Theorem. Let $M=G / H$ be a reductive semi-Riemannian homogeneous space with a fixed Lie algebra decomposition $\mathfrak{g}=\mathfrak{m}+\mathfrak{h}$ and with 1-parameter subgroups and 
connexion, $D$, as previously described so that the connexion function $\alpha(X, Y)=\frac{1}{2} X Y$ for $X, Y$ in $\mathfrak{m}$. If $M^{\prime}$ is a totally geodesic nonsingular submanifold of $M$ containing $p_{0}=H$ and if $M_{p_{0}}^{\prime}=\mathfrak{m}^{\prime} \subset \mathfrak{m}$ denotes the tangent space of $p_{0}$ in $M^{\prime}$, then $\mathfrak{m}^{\prime}$ is an abstract L.t.s. with $[X, Y, Z]$ given by (4. 7). Conversely if $\mathfrak{m}^{\prime}$ is a nonsingular subalgebra of $\mathfrak{m}$ which is an abstract L.t.s. as given by (4. 7), then there exists a totally geodesic nonsingular submanifold $M^{\prime}$ of $M=G / H$ with $p_{0}$ in $M^{\prime}$ and $M_{p_{0}}^{\prime}=\mathfrak{m}^{\prime}$.

Proof. The first part follows from statement (iv) in the proposition in section 3 concerning totally geodesic submanifolds. For the converse we note that from the remarks following (4.7) we see that the subalgebra $\mathfrak{m}^{\prime}$ is $h\left(\mathfrak{m}^{\prime}, \mathfrak{m}^{\prime}\right)$-invariant. This yields that the subspace $\mathfrak{g}^{\prime}=\mathfrak{m}^{\prime}+h\left(\mathfrak{m}^{\prime}, \mathfrak{m}^{\prime}\right)$ is actually a Lie subalgebra of $g$; for,

$$
\begin{aligned}
{\left[\mathfrak{g}^{\prime} \mathfrak{m}^{\prime}\right]=\left[\mathfrak{m}^{\prime} \mathfrak{m}^{\prime}\right]+\left[h\left(\mathfrak{m}^{\prime}, \mathfrak{m}^{\prime}\right) \mathfrak{m}^{\prime}\right] } \\
\subset \mathfrak{m}^{\prime} \mathfrak{m}^{\prime}+h\left(\mathfrak{m}^{\prime}, \mathfrak{m}^{\prime}\right)+\mathfrak{m}^{\prime} \\
\subset \mathfrak{m}^{\prime}+h\left(\mathfrak{m}^{\prime}, \mathfrak{m}^{\prime}\right)=\mathfrak{g}^{\prime}, \\
{\left[\mathfrak{g}^{\prime} h\left(\mathfrak{m}^{\prime}, \mathfrak{m}^{\prime}\right)\right]=\left[\mathfrak{m}^{\prime} h\left(\mathfrak{m}^{\prime}, \mathfrak{m}^{\prime}\right)\right] } \\
\quad+\left[h\left(\mathfrak{m}^{\prime}, \mathfrak{m}^{\prime}\right) h\left(\mathfrak{m}^{\prime}, \mathfrak{m}^{\prime}\right)\right]
\end{aligned}
$$

and this last expression is in $\mathfrak{g}^{\prime}$. To see this we use (4.5) and the assumption that $\mathfrak{m}^{\prime}$ is $h\left(\mathfrak{m}^{\prime}, \mathfrak{m}^{\prime}\right)$-invariant to obtain $\left[\mathfrak{h}\left(\mathfrak{m}^{\prime}, \mathfrak{m}^{\prime}\right) \mathfrak{h}\left(\mathfrak{m}^{\prime}, \mathfrak{m}^{\prime}\right)\right] \subset \mathfrak{h}\left(\mathfrak{m}^{\prime}, \mathfrak{m}^{\prime}\right)$. We may now proceed analogously to the case of a symmetric space. Let $G^{\prime}$ be the connected subgroup of $G$ with Lie algebra $g^{\prime}$ and let $M^{\prime}=G^{\prime} \cdot p_{0}$. Then $M^{\prime}$ is a submanifold of $M$ containing $p_{0}$ and is diffeomorphic to $G^{\prime} / K^{\prime}$ where $K^{\prime}$ is the closed subgroup of $G^{\prime}$ leaving $p_{0}$ fixed. $M_{p_{0}}^{\prime}=\mathfrak{m}^{\prime}$ so that $M^{\prime}$ is a nonsingular submanifold. Next by the assumption concerning the connexion we have that the geodesics in $M$ through $p_{0}$ are of the form $\exp t X \cdot p_{0}$ where $t$ in $R$ and $X$ in $\mathfrak{m}$. This geodesic is tangent to $M^{\prime}$ at $p_{0}$ if and only if $X \in M_{p_{0}}^{\prime}=\mathfrak{m}^{\prime}$; thus from the definition, the submanifold $M^{\prime}$ is totally geodesic at $p_{0}$. Next note that the connexion $D$ is $G^{\prime}$ invariant (because it is $G$-invariant) and $G^{\prime}$ is a group of isometries of $M$ and $M^{\prime}$ relative to the corresponding semi-Riemannian metrics (by definition of these metrics), and $G^{\prime}$ acts transitively on $M^{\prime}$. Using these we see that a geodesic at any point of $M^{\prime}$ is taken by some isometry induced by $G^{\prime}$ into a geodesic through $p_{0}$ in $M^{\prime}$ and consequently $M^{\prime}$ is geodesic at each of its points; that is, totally geodesic. 
5. Examples. Let $M=G / H$ be a semi-Riemannian reductive homogeneous space with connection function $\alpha(X, Y)=1 / 2 X Y$ as determined by the (fixed) decomposition $\mathfrak{g}=\mathfrak{m} \dot{+}$. Let $L(\mathfrak{m})$ be the Lie transformation algebra generated by all the right multiplications

$$
R(X): \mathfrak{m} \rightarrow \mathfrak{m}: Y \rightarrow Y X
$$

see [11]. Thus $L(\mathfrak{m})=M_{1}+M_{2}+\cdots$ where $\quad M_{1}=\{R(X): X \in \mathfrak{m}\} \quad$ and $M_{k}=\left[M_{1}, M_{k-1}\right]$.

The holonomy algebra, $\mathfrak{b}^{*}$, which is the Lie algebra of the holonomy group of $G / H$ relative to the connection given by $\alpha(X, Y)=1 / 2 X Y$ is the smallest Lie algebra of endomorphisms of $\mathfrak{m}$ such that $(1)\left[R(X), \mathfrak{h}^{*}\right] \subset \mathfrak{h}^{*}$ and (2) the curvature endomorphism $R(X, Y) \in \mathfrak{h}^{*}$ for all $X, Y \in \mathfrak{m}$ and $R(X, Y)$ is given by (4. 7). When $\mathfrak{m}$ is a simple algebra (with multiplication $X Y$ and nondegenerate invariant form $\langle X, Y\rangle$ ) it can be shown that the derivations $D(h(X, Y))=a d_{\mathfrak{m}} h(X, Y) \in L(\mathfrak{m})$. Using this we have the following result $[10]$.

Theorem. Let $G / H$ be a simply connected semi-Riemannian reductive homogeneous space with connection function $\alpha(X, Y)=1 / 2 X Y$ determined by the fixed decomposition $\mathfrak{g}=\mathfrak{m}+\mathfrak{h}$. Assume $G / H$ is not symmetric (i.e. $\mathfrak{m m} \neq 0)$. Then $G / H$ is holonomy irreducible if and only if $\mathfrak{m}$ is a simple algebra. Furthermore the holonomy algebra $\mathfrak{h}^{*}=L(\mathfrak{m})$ and is semi-simple.

Remarks. In case $M=G / H$ is Riemannian, the deRham decomposition theorem can be used to show $M=S \times M^{\prime}$ with $\mathfrak{m}=\mathfrak{i} \oplus \mathfrak{m}^{\prime}$ where $\mathfrak{i m ^ { \prime }}=\mathfrak{i f}=0$ and $\mathfrak{i}$ corresponds to the symmetric space part $S$ of $M$ and $\mathfrak{m}^{\prime}=\mathfrak{m}_{1}^{\prime} \oplus \cdots$ $\oplus \mathfrak{m}_{r}^{\prime}$ is a direct sum of simple ideals which corresponds to the nonsymmetric space part $M^{\prime}$ of $M$.

If the nondegenerate form $\langle X, Y\rangle$ is such that there are no ideals $\mathfrak{n}$ of $\mathfrak{m}$ such that $\mathfrak{n}^{2}=0$, then from $\left[4\right.$, p. 71] we have $\mathfrak{m}=\mathfrak{m}_{1} \oplus \cdots \oplus \mathfrak{m}_{r}$ where $\mathfrak{m}_{j}$ are simple ideals. For example if $\mathfrak{m}$ is a Lie or Malcev algebra, the form $\langle X, Y\rangle=$ trace $R(X) R(Y)$ works.

The nondegenerate invariant symmetric forms on a simple anticommutative algebra need not be unique. But if $\langle X, Y\rangle$ and $\langle X, Y\rangle^{\prime}$ are two such forms, then these differ by a multiple in the centroid of $M$ [4, p. 290]. That is, there exists a unique linear transformation $A$ in 
$\Gamma(\mathfrak{m})=\{T \in \operatorname{Hom}(\mathfrak{m}, \mathfrak{m}):[T, R(X)]=0$ all $X \in \mathfrak{m}\}$ such that $\langle X, Y\rangle=\langle X, A Y\rangle^{\prime}$ for all $X, Y$ in $\mathfrak{m}$. The proof of this is as in the case of Lie algebras; for a fixed $Y$ in $\mathfrak{m}$ the form $\langle X, Y\rangle$ is a linear functional on $\mathfrak{m}$ and since $\langle X, Y\rangle^{\prime}$ is nondegenerate, there exists a unique $Y^{\prime}$ in $\mathfrak{m}$ such that $\langle X, Y\rangle=\left\langle X, Y^{\prime}\right\rangle^{\prime}$ for all $X$ in $\mathfrak{m}$. The unique map $A: \mathfrak{m} \rightarrow \mathfrak{m}: Y \rightarrow Y^{\prime}$ is a linear transformation; thus $\langle X, Y\rangle=\langle X, A Y\rangle^{\prime}$ for all $X, Y$ in $\mathfrak{m}$. Next $\quad\langle X Y, Z\rangle=\langle X Y, A Z\rangle^{\prime}=\langle X, Y(A Z)\rangle^{\prime}$; but $\quad\langle X Y, Z\rangle=\langle X, Y Z\rangle$ $=\langle X, A(Y Z)\rangle^{\prime}$. Since the forms are nondegenerate, $Y(A Z)=A(Y Z)$ which implies $[A, R(Y)]=0$; that is $A$ is in $\Gamma(\mathfrak{m})$. This proof holds for any field, however over the reals we have that $\Gamma(\mathfrak{m})=R I$ or $\Gamma(\mathfrak{m})=R I+R J$ which is isomorphic to the complex numbers where $J^{2}+I=0[4$, p. 298] Thus in this case $A=\alpha I+\beta J$ and also $\langle A X, Y\rangle=\langle X, A Y\rangle$.

The preceding has an obvious extension to the case when $\mathfrak{m}=\mathfrak{m}_{1} \oplus \cdots$ $\oplus \mathfrak{m}_{k}$ where the $\mathfrak{m}_{i}$ are simple ideals. Since $\mathfrak{m}_{i}=\mathfrak{m}_{i}^{2}$ the restriction of $\langle X, Y\rangle$ to $\mathfrak{m}_{i}$ is a nondegenerate invariant form; in particular for $X=\Sigma X_{i}, Y=\Sigma Y_{i}$ in $\mathfrak{m},\left\langle X, Y>=\Sigma<X_{j}, Y_{j}>\right.$. Thus if $\langle X, Y\rangle^{\prime}$ is any other non-degenerate form on $\mathfrak{m}, \quad<X_{i}, Y_{i}>^{\prime}=<X_{i}, A_{i} Y_{i}>$ where $A_{i}$ is a unique element of $\Gamma\left(\mathfrak{m}_{i}\right)$ and therefore $\left.\left.<X, Y\right\rangle^{\prime}=\Sigma<X_{i}, A_{i} Y_{i}\right\rangle$. For example these remarks apply to compact Lie groups as follows.

If $G$ is compact, then the Killing form $B(P, Q)$ of $g$ is negative definite. Thus if $\mathfrak{h}$ is the Lie algebra of $H$ we may write $\mathfrak{g}=\mathfrak{m}+\mathfrak{h}$ with $\mathfrak{m}=\mathfrak{h} \perp$ relative to $B(P, Q)$. The form $B(P, Q)$ restricted to $\mathfrak{m}$ is a nondegenerate invariant form. For if $X$ is in $\mathfrak{m}$ and $B(X, \mathfrak{m})=0$, then since $\mathfrak{m}=\mathfrak{h} \perp$ we have $B(X, \mathfrak{h})=0$ so that $B(X, \mathfrak{g})=0$; thus $X=0$. Also for $X, Y, Z$ in $\mathfrak{m}$, $B(X Y, Z)=B(X Y+h(X, Y), Z)=([X Y], Z)=B(X,[Y Z])=B(X, Y Z)$. Next if $\mathfrak{t}$ is an ideal of $\mathfrak{m}$ with $\mathfrak{t}^{2}=0$, then $B(\mathfrak{n} \mathfrak{m}, \mathfrak{n} \mathfrak{m}) \subset B(\mathfrak{i t}, \mathfrak{n} \mathfrak{m})=B\left(\mathfrak{t}^{2}, \mathfrak{m}\right)=0$ and since $B(P, Q)$ is definite on $\mathfrak{m}$, we have $\mathfrak{i t m}=0$. From this $\mathfrak{i}=\sum \mathfrak{n}$, summed over all ideals $\mathfrak{n}$ with $\mathfrak{n}^{2}=0$, is an ideal of $\mathfrak{m}$ such that $\mathfrak{f}^{2}=\sum \mathfrak{i} t$ $=0$. Thus by the deRham decomposition theorem $\mathfrak{m}=\mathfrak{i} \oplus \mathfrak{m}^{\prime}$ where $\mathfrak{m}^{\prime}=\mathfrak{m}_{1}^{\prime} \oplus \cdots \oplus \mathfrak{m}_{r}^{\prime}$ is a direct sum of simple ideals and $-B(P, Q)$ restricted to $\mathfrak{m}_{j}$ yields a positive definite form $B_{j}(X, Y)$ on $\mathfrak{m}_{j}$. Thus if $\langle X, Y\rangle_{j}$ is any other nondegenerate invariant form on $\mathfrak{m}_{j}$, then for $X, Y$ in $\mathfrak{m}_{j}$ we have $<X, Y\rangle_{j}=B_{j}\left(A_{j} X, Y\right)$ with $A_{j}$ uniquely determined in $\Gamma\left(\mathfrak{m}_{j}\right)$ : But $A_{j}=\alpha_{j} I+\beta_{j} J$ and is self-adjoint relative to $B_{j}(X, Y)$. Thus if $J$ exists in $\Gamma\left(\mathfrak{m}_{j}\right)$, then $J$ has a symmetric matrix and therefore a real characteristic root $\lambda$ satisfying $\lambda^{2}+1=0$; so $J$ does not exist. Thus $\langle X, Y\rangle_{j}=\alpha_{j} B_{j}(X, Y)$ 
is uniquely determined on each $\mathfrak{m}_{j}$ and thus uniquely determined on the non-symmetric space part of $G / H$.

The two forms trace $R(X) R(Y)$ and $B(X, Y)$ just discussed are closely related even if they are not nondegenerate or invariant; essentially the difference between these forms is the Ricci scalar curvature of $M=G / H$. First let $\sigma(X, Y): \mathfrak{m} \rightarrow \mathfrak{m}: Z \rightarrow[h(X, Z) Y]$ and let $s(X, Y)=\operatorname{trace} \sigma(X, Y)$. Then from (4. 3),

$$
[R(Y), R(X)]-R(Y X)=\sigma(Y, X)-\sigma(X, Y)+D(h(X, Y))
$$

and since trace $R(Z)=0=\operatorname{trace} D$ we have $s(X, Y)=s(Y, X)$. Next from (4. 7) we have that the mapping

$$
\rho(X, Y): \mathfrak{m} \rightarrow \mathfrak{m}: Z \rightarrow R(X, Z) Y
$$

is given by $\rho(X, Y)=-\frac{1}{4} R(X) R(Y)-\frac{1}{4} R(X Y)+\frac{1}{2} R(Y) R(X)-\sigma(X, Y)$. Thus the Ricci scalar curvature [1] of $M$ is $r(X, Y)=$ trace $\rho(X, Y)=\frac{1}{4}$ trace $R(X) R(Y)-s(X, Y)$. This form is symmetric since $s(X, Y)$ is symmetric. Now a straightforward calculation of the Killing form in $\mathfrak{g}$ show that for $X, Y$ in $\mathfrak{n}$

$$
\begin{aligned}
B(X, Y) & =\text { trace } a d_{\mathfrak{g}} X a d_{\mathfrak{g}} Y \\
& =\frac{1}{2} \text { trace } R(X) R(Y)+2 r(X, Y)
\end{aligned}
$$

We shall now give some examples of reductive spaces in terms of the simple algebra $\mathfrak{m}$ for which $B(X, Y)$ is a multiple of trace $R(X) R(Y)$ and for which totally geodesic submanifolds are easily determined. From the theorem in the first part of this section we see that the derivations $D(h(X, Y))$ are essentially polynomials in elements from $\{R(Z): Z$ in $\mathfrak{m}\}$. We now consider the case where there is a well behaved formula for each $D(h(X, Y))$ in terms of elements from the Lie subalgebra $L(X, Y)$ which is generated by $\{R(Z): Z$ in $\mathfrak{m}(X, Y)\}$ where $\mathfrak{m}(X, Y)$ is the subalgebra of $\mathfrak{m}$ generated by $X, Y$ in $\mathfrak{m}$. Thus we are assuming there exists an ordered family of functions

$$
A(\mathfrak{m})=\left\{a_{0}, a_{1}, a_{2} ; b_{i} \text { with } i=1, \cdots, 5 ; c_{i_{1}} \ldots i_{k}\right\}
$$

which depend only on $\mathfrak{m}$ and are of class $C^{1}(\mathfrak{m} \times \mathfrak{m})$, regarding $\mathfrak{m}$ as the usual Euclidean vector space, and such that for every $X, Y$ in $\mathfrak{m}$, 
(5. 1)

$$
\begin{aligned}
D(h(X, Y)) & =a_{0}(X, Y) I+a_{1}(X, Y) R(X)+a_{2}(X, Y) R(Y) \\
& +b_{1}(X, Y) R(X) R(Y)+b_{2}(X, Y) R(Y) R(X) \\
& +b_{3}(X, Y) R(X Y)+b_{4}(X, Y) R(X)^{2}+b_{5}(X, Y) R(Y)^{2} \\
& +\sum c_{i_{1}} \cdots i_{k}(X, Y) R\left(Z_{i_{1}}\right) \cdots R\left(Z_{i_{k}}\right)
\end{aligned}
$$

where the $Z_{i_{1}}, \cdots, Z_{i_{h}}$ in $\mathfrak{m}(X, Y)$ are determined by the $c_{i_{1}} \ldots i_{k}(X, Y)$ and the terms in the sum $\Sigma$ are homogeneous in $X$ and $Y$ of degree $\geq 3$. Using (5.1) and a few of the identities for the simple algebra $\mathfrak{m}$ we shall show

$$
\begin{aligned}
D(h(X, Y))=f_{0}(X, Y) I & +f(Y) R(X)-f(X) R(Y) \\
& +b_{1}[R(X), R(Y)]+b_{3} R(X Y)
\end{aligned}
$$

where $f_{0}$ is bilinear on $\mathfrak{m}, f$ is a linear functional and $b_{1}, b_{3}$ are in $R$.

Remarks. Since $L(X, Y)$ is finite dimensional the right side of equation (5. 1) is defined; the set of functions $A(\mathfrak{m})$ is used to determine the elements from $L(X, Y)$ for which the functions in $A(\mathfrak{m})$ are to be the coefficients. This formulation is the same as assuming an identity $D(h(X, Y)) Z=a_{0}(X, Y) Z+\cdots$ given by (5. 1) with the functions defined by the identity, say $a_{0}: \mathfrak{m} \times \mathfrak{m}$ $\rightarrow R:(X, Y) \rightarrow a_{0}(X, Y)$, of class $C^{1}(\mathfrak{m} \times \mathfrak{m})$.

We now determine (5.2). Let $t$ be in $R$, then for $D(X, Y) \equiv D(h(X, Y))$ we have $t D(X, Y)=D(t X, Y)$

$$
\begin{aligned}
& =a_{0}(t X, Y) I+a_{1}(t X, Y) R(t X)+a_{2}(t X, Y) R(Y) \\
& +b_{1}(t X, Y) R(t X) R(Y)+b_{2}(t X, Y) R(Y) R(t X) \\
& +b_{3}(t X, Y) R(t X Y)+b_{4}(t X, Y) R(t X)^{2} \\
& +b_{5}(t X, Y) R(Y)^{2} \\
& +\sum c_{i_{1}} \ldots i_{k}(t X, Y) R\left(Z_{i_{1}}(t)\right) \cdots R\left(Z_{i_{k}}(t)\right)
\end{aligned}
$$

where $Z_{i_{j}}(t)$ are in $\mathfrak{m}(X, Y)$. Now note that if the expression $R\left(Z_{i_{1}}\right) \cdots R\left(Z_{i_{k}}\right)$ is of degree $>1$ in $X$, then

$$
\frac{d}{d \bar{t}} R\left(Z_{i_{1}}(t)\right) \cdots R\left(Z_{i_{k}}(t)\right)=0
$$

at $t=0$. Thus using the product rule of differentiation,

$$
\begin{aligned}
D(X, Y) & \left.=\frac{d(t D}{d t}(X, Y)\right), \text { at } t=0 \\
& =\left[g_{0}(Y)\right](X) I+a_{1}(0, Y) R(X) \\
& +\left[g_{2}(Y)\right](X) R(Y)+b_{1}(0, Y) R(X) R(Y)
\end{aligned}
$$




$$
\begin{aligned}
& +b_{2}(0, Y) R(Y) R(X)+b_{3}(0, Y) R(X Y) \\
& +b_{5}(0, Y) R(Y)^{2} \\
& +\sum \bar{c}_{i_{1}} \ldots i_{k} R\left(W i_{1}\right) \cdots R\left(W i_{k}\right)
\end{aligned}
$$

where each term $R\left(W_{i_{1}}\right) \cdots R\left(W_{i_{k}}\right)$ is of degree $\leq 1$ in $X$ and of degree $\geq 2$ in $Y$ and $\left[g_{j}(Y)\right](X)=d a_{j}(t X, Y) / d t$ at $t=0$ defines a linear functional $g_{j}(Y)$ on $\mathfrak{m}$. Similarly, using this last expression for $D(X, Y)$ and differentiating $t D(X, Y)=D(X, t Y)$ at $t=0$ we obtain

$$
\begin{aligned}
D(X, Y) & =f_{0}(X, Y) I+f_{1}(Y) R(X)+f_{2}(X) R(Y) \\
& +b_{1} R(X) R(Y)+b_{2} R(Y) R(X)+b_{3} R(X Y)
\end{aligned}
$$

using $\Sigma$ is homogeneous of degree $\geq 2$ in $Y$ and where $b_{i}=b_{i}(0,0)$ is in $R, \quad f_{1}(Y)=d a_{1}(0, t Y) / d t \quad$ at $t=0$ and $f_{2}(X)=\left[g_{2}(0)\right](X)$ define linear functionals on $\mathfrak{m}$, and $f_{0}(X, Y)=d\left[g_{0}(t Y)\right](X) / d t$ at $t=0$ defines a bilinear function on $\mathrm{mt}$.

Next we have $0=D(X, X)=f_{0}(X, X) I+\left(f_{1}+f_{2}\right)(X) R(X)+\left(b_{1}+b_{2}\right) R(X)^{2}$ so that applying this to $X$ we obtain $f_{0}(X, X)=0$ for all $X$ in $\mathfrak{m}$. Applying the resulting formula for $D(X, X)=0$ to any $Y$ in $\mathfrak{m}$ we obtain

$$
\left[-\left(f_{1}+f_{2}\right)(X)\right] Y X=\left(b_{1}+b_{2}\right)(Y X) X
$$

and linearizing

$$
\left[-\left(f_{1}+f_{2}\right)(X)\right] Y Z+\left[-\left(f_{1}+f_{2}\right)(Z)\right] Y X=\left(b_{1}+b_{2}\right)[(Y X) Z+(Y Z) X] .
$$

Now since we are assuming $\mathfrak{m}$ is a simple finite dimensional algebra, $\operatorname{dim} . \mathfrak{m}>1$ and therefore there exists $P \neq 0$ in $\mathfrak{m}$ with $\left(f_{1}+f_{2}\right)(P)=0$ (using kernel of linear functional $\neq 0)$. Let $\mathfrak{p}=P_{\mathfrak{m}}$, then

$$
\begin{aligned}
\left(b_{1}+b_{2}\right)(Y P) Z & =-\left(b_{1}+b_{2}\right)(Y Z) P \\
& +\left[-\left(f_{1}+f_{2}\right)(Z)\right] Y P
\end{aligned}
$$

which is in $\mathfrak{p}$ and shows $\mathfrak{p}$ is an ideal of $\mathfrak{m}$ if $b_{1}+b_{2} \neq 0$. Now if $\mathfrak{p}=\mathfrak{m}$, then $R(P)$ is surjective and therefore injective; but $R(P) P=0$. This contradiction shows $\mathfrak{p}=0$ which implies $P R$ is a one dimensional ideal which must equal $\mathfrak{m}$. This contradiction yields $b_{1}+b_{2}=0$. From this we have $\left(f_{1}+f_{2}\right)(X) R(X)=0$ for all $X$ in $\mathfrak{m}$ and this implies $f_{1}+f_{2}=0$ which proves (5.2).

We now determine completely the above algebras $\mathfrak{m}$. An algebra $\mathfrak{m}$ obtained from a Lie algebra decomposition $\mathfrak{g}=\mathfrak{m}+\mathfrak{h}$ is called flat if $\mathfrak{m}$ not only satisfies (4. 1)-(4.6) but also $R(X, Y) Z=0$ in (4.7). 
Theorem. Let $\mathfrak{m}$ be the algebra obtained from a Lie algebra decomposition $\mathfrak{g}=\mathfrak{m}+\mathfrak{h}$ with $[\mathfrak{m}, \mathfrak{h}] \subset \mathfrak{m}$. If $\mathfrak{m}$ is a simple Lie, Malcev or flat algebra, then it satisfies (4. 1)-(4.6) and its derivations are given by (5.2) with $f_{0} \equiv f \equiv 0$. Conversely if $\mathfrak{m}$ is a simple algebra as has been discussed; that is, $\mathfrak{m}$ satisfies (4. 1)-(4.6) and has a nondegenerate invariant form $\langle X, Y\rangle$ such that $\langle D X, Y\rangle$ $+<X, D Y>=0$ and $\langle X Y, Z>=<X, Y Z>$ for all $D=D(U, V)$ and $X, Y, Z, U, V \in \mathfrak{m}$, and $\mathfrak{m}$ also satisfies the condition discussed in (5.1), then $\mathfrak{m}$ is a Lie, Malcev or flat algebra. In these respective cases we have $b_{1}-b_{3}=1$ and $D(X, Y) Z=(X Y) Z ;-b_{1}=b_{3}=1$ and $D(X, Y) Z=-(X Y) Z+(Y Z) X+(Z X) Y$ (and $\mathfrak{m}$ not a Lie algebra); $2 b_{1}=b_{3}=\frac{1}{2}$ and $D(X, Y) Z=\frac{1}{2}\left[(X Y) Z+\frac{1}{2}(Y Z) X\right.$ $\left.+\frac{1}{2}(Z X) Y\right]$.

Proof. If $\mathfrak{m}$ is Lie, Malcev or flat, then the results follow from [7] where the formulas for the derivations are also determined. For the converse we first note that from the assumption concerning the invariant form trace $[R(X)]=\operatorname{trace} D=0$ so that from (5.2), $0=\operatorname{trace} D(X, Y)=f_{0}(X, Y)$ (trace I); therefore $f_{0}(X, Y)=0$. We now rewrite (4.3)-(4.6) using (5.2) with $f_{0}=0$ to obtain from (4.3)

$$
\text { (5. 3) } \begin{aligned}
\left(2 b_{1}+b_{3}-1\right) J(X, Y, Z) \\
=2[f(X) Y Z+f(Y) Z X+f(Z) X Y] .
\end{aligned}
$$

For $D=D(h): X \rightarrow\left[\begin{array}{ll}h & X\end{array}\right]$ we have from (4.5) that $[D(h), D(X, Y)]$ $=D(D(h) X, Y)+D(X, D(h) Y)$ and using (5.2) in this expression we eventually obtain

$$
f(D(h) X) R(Y)=f(D(h) Y) R(X) .
$$

Now since $\mathfrak{m}$ is simple, (5.4) implies $f(D(h) X) Y=f(D(h) Y) X$; otherwise there exists $A \neq 0$ in $\mathfrak{m}$ with $R(A)=0$ which implies that $\mathfrak{m}$ has a one dimensional ideal. But since dim. $\mathfrak{m}>1$ this formula implies $f(D(h) X)=0$ for all $X$ in $\mathfrak{m}$ and $h$ in $\mathfrak{h}$. For $D(h)$ of the form $D(X, Y)$ we have from (4. 3),

$$
f(J(X, Y, X))=0 .
$$

But from (5. 2) we also have

$$
\begin{aligned}
0 & =f(D(X, Y) U) \\
& =f(f(Y) U X-f(X) U Y) \\
& +f\left(-b_{1} J(X, Y, U)+\left(b_{3}-b_{1}\right) U(X Y)\right) ;
\end{aligned}
$$


which implies

$$
f(Y) f(U X)+f(X) f(Y U)=\left(b_{1}-b_{3}\right) f(U(X Y)) .
$$

Using this and (5.3) we have

$$
\begin{aligned}
0 & =\frac{1}{2}\left(2 b_{1}+b_{3}-1\right) f(J(X, Y, U)) \\
& =f(X) f(Y U)+f(Y) f(U X)+f(U) f(X Y) \\
& =\left(b_{1}-b_{3}\right) f(U(X Y))+f(U) f(X Y) .
\end{aligned}
$$

Set $U=X Y$ in this last equation to obtain $f(X Y)=0$ for all $X, Y$ in $\mathfrak{m}$. But since $\mathfrak{m}$ is simple $\mathfrak{m}=\mathfrak{m}^{2}$ which implies $f \equiv 0$ on $\mathfrak{m}$ and the results now follow from [7].

Thus the algebras $\mathfrak{m}$ satisfying (5.1) are such that a subalgebra $\mathfrak{m}^{\prime}$ is $\mathfrak{h}\left(\mathfrak{m}^{\prime}, \mathfrak{m}^{\prime}\right)$-invariant so that using the remarks following (4.7) we see that these algebras $\mathfrak{m}$ are such that nonsingular subalgebras yield totally geodesic submanifolds according to the theorem in section 4. Putting these algebras into their reductive context, $\mathfrak{g}=\mathfrak{m} \dot{+} \mathfrak{h}$, a straightforward computation using (5. 2) shows $s(X, Y)=$ trace $\sigma(X, Y)=\left(b_{3}-b_{1}\right)$ trace $R(X) R(Y)$; consequently the Killing form on $\mathfrak{m}$ and the Ricci scalar curvature are $B(X, Y)=(1+$ $\left.2 b_{1}-2 b_{3}\right)$ trace $R(X) R(Y)$ and $r(X, Y)=\left(\frac{1}{4}+b_{1}-b_{3}\right)$ trace $R(X) R(Y)$.

For simple Lie and Malcev algebras $\langle X, Y\rangle=$ trace $R(X) R(Y)$ is known to be a nondegenerate invariant form and consequently define an essentially unique metric on the corresponding homogeneous space $M=G / H$ (e.g., $G$ is of type $B_{3}, H$ of type $G_{2}$ in the Malcev case). If $m$ is flat the form $<X, Y>$ satisfies $<X Y, Z>+<Y Z, X>+<Z X, Y>=-3$ trace $[R(X), R(Y)] R(Z)$ and the identities or simplicity do not appear to imply $\langle X, Y\rangle$ is invariant.

\section{REFERENCES}

[1] S. Helgason: "Differential Geometry and Symmetric Spaces", Academic Press, 1962.

[2] N. Hicks: Submanifolds of semi-Riemannian Manifolds, Rend. Cir. Mat. Palermo, Vol. 12 (1964), 1-13.

[ 3 ] N. Hicks: "Notes on Differential Geometry", Van Nostrand, 1965.

[4] N. Jacobson: "Lie Algebras", Interscience, 1962.

[ 5 ] W.G. Lister: A structure theory of Lie triple systems, Trans. Amer. Math. Soc., Vol. 72 (1952), 217-242.

[6] K. Nomizu: Invariant affine connections on homogeneous spaces, Amer. J. Math., Vol. 76 (1954), 33-65. 
[ 7 ] A. Sagle: On anti-commutative algebras and general Lie triple systems, Pacific J. Math., Vol. 15 (1965), 281-291.

[8] A. Sagle: On anti-commutative algebras with an invariant form, Canadian Jol. Math., Vol. 16 (1964), 370-378.

[9] A. Sagle: On simple algebras obtained from general Lie triple systems, to appear Pacific Jol. Math.

[10] A. Sagle: On anti-commutative algebras and homogeneous spaces, to appear in Jol. Math. and Mechanics.

[11] R.D. Schafer: Inner derivations of nonassociative algebras, Bull. Amer. Math. Soc., Vol. 55 (1949), 769-776.

[12] I. Singer: Differential Geometry, M.I.T. notes (1962).

[13] K. Yamaguti: On algebras of totally geodesic spaces (Lie triple systems), Jol. Hiroshima Univ., Vol. 21 (1957), 107-113.

University of Minnesota 